\title{
A SYNTHETIC APERTURE IMAGING SYSTEM USING SURFACE WAVE MODES
}

\author{
A. Bozkurt, O. Arıkan, A. Atalar \\ Electrical and Electronics Engineering Department \\ Bilkent University, 06533 Bilkent, Ankara, TURKEY
}

\section{Abstract}

A synthetic aperture acoustic imaging system with a novel inversion algorithm is described. Data is obtained by using a transducer insonifying the sample surface at a critical angle which is excited by a short electrical pulse. The critical angle is chosen for a suitable surface wave or Lamb wave mode that exists on the object. The transducer is mechanically scanned in only one direction during which many pulse excitations and subsequent recordings are realized. The received signal is sampled in time and digitized to be processed by using the new inversion approach providing an optimal $2-\mathrm{D}$ image of the surface reflectivity.

\section{Introduction}

Acoustic synthetic aperture imaging has found many application areas. Various inversion algorithms have been proposed to obtain high resolution images $[1,2]$. In this report, a new inversion algorithm is proposed to obtain high resolution images from reflection data acquired in a measurement geometry shown in Fig. 1. In this geometry, the measurements are obtained by using a transducer exited by a short electrical pulse. The transducer insonifies the sample surface at a critical angle which is chosen for a suitable surface wave or Lamb wave mode that exists on the object. The transducer is mechanically scanned in only one direction during which many pulse excitations and subsequent recordings are realized. Although the data acquisition scenario has similarities to that of Synthetic Aperture Radar (SAR), there are significant differences between them. Since, the scan path of the transducer is relatively closer to the surface patch of interest, the wavefront curvature is more prominent. Also, the transmitted pulses are not necessarily the same. Hence, the available efficient inversion techniques for SAR measurements need major modification to be applicable in this case.

In this work, we begin with a very accurate forward modeling of the data acquisition. Then, by using Singular Value Decomposition (SVD) of the measurement kernel, we optimally reduced a single measurement of both time and space to multiple measurements of space only. This reduction not only provides noise immunity, but also leads to a specific integral equation form for which an efficient inversion algorithm has been reported [3]. The inversion is performed in two stages. First, the measurements are filtered by using multichannel deconvolution filters. Then the results are used to weight the set of vectors that define the measurable subspace of the surface properties. Since both these vectors and the set of multichannel deconvolution filters are just functions of the data acquisition geometry, they can be precomputed to provide efficiency to the repeated use of this inversion approach.

\section{Measurement Model}

The forward model proposed for the measurement system assumes that a point scatterer on the object surface produces a transducer output proportional to the square of the field amplitude at that point [6]. Hence, the response of a point scatterer at $(\mathrm{x}, \mathrm{y})$ is

$$
K(x, y, t)=\left|u_{S A W}(x, y)\right|^{2} P\left(t-2 \sqrt{x^{2}+y^{2}} / V_{\mathrm{R}}\right)
$$

where $P$ is the transmitted RF-pulse, and $V_{\mathrm{R}}$ is the SAW velocity for the object material. The time 
waveform measured at the transducer is given by

$$
g(x, t)=\int_{-\infty}^{+\infty} \int_{0}^{+\infty} K\left(x-x^{\prime}, y, t\right) p\left(x^{\prime}, y\right) d y d x .
$$

where $p(x, y)$ describes surface objects. The field expression on the object surface is found numerically. The field at the transducer surface is propagated using angular spectrum decomposition.

\section{Inversion Algorithm}

In this section, the measurement model of the data acquisition geometry shown in Fig. 1 will be put into a new form which allows us to use efficient inversion algorithms that are developed for a class of such measurements. We begin with the measurement relation

$$
g(x, t)=\int_{-\infty}^{\infty} \int_{0}^{\infty} K\left(x-x^{\prime}, y, t\right) p\left(x^{\prime}, y\right) d x^{\prime} d y .
$$

The near vicinity of the transducer contributes most of the energy in the measurements. Hence, with a judicious choice of $\Delta_{x}$ and $\Delta_{y}$, this measurement relation can be well approximated by

$g(x, t)=\int_{x-\Delta_{x}}^{x+\Delta_{x}} \int_{0}^{\Delta_{y}} K\left(x-x^{\prime}, y, t\right) p\left(x^{\prime}, y\right) d x^{\prime} d y$.

The transducer acquires data along $x$-axis at locations $\delta_{x}$ apart, which is roughly the expected resolution cell size along the $x$-axis. However, the size of the resolution cell along the $y-$ axis gets larger for larger values of $y$. Therefore, the integrals of the measurement model should be approximated with a non-uniform 2-D Riemann sum approximation, giving

$$
\begin{aligned}
& g\left(n \delta_{x}, t\right)= \\
& \sum_{n^{\prime}=n-N}^{n+N} \sum_{l=1}^{L} K\left(\left(n-n^{\prime}\right) \delta_{x}, y_{l}, t\right) \delta_{x} \delta_{y_{l}} p\left(n^{\prime} \delta_{x}, y_{l}\right)
\end{aligned}
$$

where along the $y$-axis $L$ non-uniform partitions with size $\delta_{y_{l}}$ are chosen. The decision on the size of this non-uniform partition is based on the sensitivity of the measurements to a reflector located at various positions along the $y$-axis. The cell size is chosen larger when the sensitivity gets lower. Such non-uniform partitions have been used before including [3]. In this new form of the measurement relation the the effective measurement kernel can be identified as:

$$
K_{e}(n, l, t)=K\left(n \delta_{x}, y_{l}, t\right) \delta_{x} \delta_{y_{l}} .
$$

The following auxiliary function will be used to obtain the final form of the measurement relation:

$$
Q\left(t_{1}, t_{2}\right)=\sum_{n=-N}^{N} \sum_{l=1}^{L} K_{e}\left(n, l, t_{1}\right) K_{c}\left(n, l, t_{2}\right) \text {. }
$$

Let the Hilbert-Schmidt decomposition of this auxiliary function be:

$$
Q\left(t_{1}, t_{2}\right)=\sum_{m=1}^{\infty} \sigma_{m} q_{m}\left(t_{1}\right) q_{m}\left(t_{2}\right)
$$

where $\sigma_{1} \geq \sigma_{2} \geq \ldots \geq 0$ are called the singular values, and $q_{m}(t)$ 's are called the singular functions which form an orthonormal set of functions. An accurate approximation to this decomposition can be obtained by using the Singular Value Decomposition (SVD) of the discretized form of the $Q\left(t_{1}, t_{2}\right)$ function $[4,5]$. The singular values obtained with such a discretization is shown in Fig. 2. As seen in this figure, only the first few of singular values dominates the rest providing the following approximation:

$$
Q\left(t_{1}, t_{2}\right) \approx \sum_{m=1}^{M} \sigma_{m} q_{m}\left(t_{1}\right) q_{m}\left(t_{2}\right)
$$

Now using the obtained singular functions, the effective kernels can be rewritten as:

$$
K_{\varepsilon}(n, l, t)=\sum_{m=1}^{M} K_{m}(n, l) q_{m}(t) .
$$

This leads to the following approximation to the measurement relation:

$$
\begin{aligned}
& g\left(n \delta_{x}, t\right)= \\
& \sum_{n^{\prime}=n-N}^{n+N} \sum_{l=1}^{L} \sum_{m=1}^{M} K_{m}\left(n-n^{\prime}, l\right) q_{m}(t) p\left(n^{\prime} \delta_{x}, y_{l}\right)
\end{aligned}
$$

Now, by using the orthonormality of the singular functions, $q_{m}(t)$ 's, we get the following set of relations for $1 \leq m \leq M$ :

$$
\begin{aligned}
g_{m}(n) & =\int_{0}^{\infty} g\left(n \delta_{x}, t\right) q_{m}(t) d t \\
& =\sum_{n^{\prime}=n-N}^{n+N} \sum_{l=1}^{L} K_{m}\left(n-n^{\prime}, l\right) p\left(n^{\prime}, l\right)
\end{aligned}
$$


where $p\left(n^{\prime}, l\right)=p\left(n^{\prime} \delta_{x}, y_{l}\right)$. This final form of the measurement relation replaces the single space and time measurement of $g(x, t)$ with $M$ measurements space measurements $g_{m}(n)$. In doing so, we also eliminate that part of the inevitable additive measurement noise which is not in the span of the $q_{m}(t)$ functions. A regularized inversion method for this type of measurement relations has been investigated at depth in [3]. In the rest of this section, major points of the inversion method will be presented.

The final form of the measurement relation

$$
\begin{array}{r}
g_{m}(n)=\sum_{n^{\prime}=n-N}^{n+N} \sum_{l=1}^{L} K_{m}\left(n-n^{\prime}, l\right) p\left(n^{\prime}, l\right) \\
1 \leq m \leq M
\end{array}
$$

has the form of convolution and projection operations. This form can be further exploited by using the SVD of the concatenated kernel matrix:

$$
\mathbf{K}=\left[\begin{array}{llll}
\mathbf{K}_{1}^{\mathbf{T}} & \mathbf{K}_{2}^{\mathbf{T}} & \ldots & \mathbf{K}_{\mathbf{M}}^{\mathbf{T}}
\end{array}\right]^{\mathbf{T}}
$$

where $^{\mathbf{T}}$ denotes the transpose operator. The SVD of $\mathbf{K}$ can be computed to obtain:

$$
\mathbf{K}=\mathbf{U S V}^{\mathbf{T}}
$$

where $\mathbf{U}$ and $\mathbf{V}$ have orthonormal columns, and $\mathbf{S}$ is a square diagonal matrix with positive nonincreasing diagonal entries [5]. The matrix $\mathbf{W}=$ US can be partitioned into equal sized matrices $\mathbf{W}_{\mathrm{m}}$ for $1 \leq m \leq M$ such that:

$$
\mathbf{W}=\left[\begin{array}{llll}
\mathbf{W}_{\mathbf{1}}^{\mathbf{T}} & \mathbf{W}_{\mathbf{2}}^{\mathbf{T}} & \ldots & \mathbf{W}_{\mathbf{M}}^{\mathbf{T}}
\end{array}\right]^{\mathbf{T}} .
$$

Now, let $\mathbf{w}_{\text {mi }}$ for $1 \leq i \leq I$ be the $i^{\text {th }}$ column of $\mathbf{W}_{\mathbf{m}}$. Then, the measurement relation can be rewritten as:

$$
\begin{aligned}
& g_{m}(n)= \\
& \sum_{n^{\prime}=n-N}^{n+N} \sum_{i=1}^{L} \sum_{i=1}^{I} w_{m i}\left(n-n^{\prime}\right) v_{i}(l) p\left(n^{\prime}, l\right), \\
& 1 \leq m \leq M
\end{aligned}
$$

which can be regrouped to obtain:

$$
\begin{array}{r}
g_{m}(n)=\sum_{i=1}^{I} \sum_{n^{\prime}=n-N}^{n+N} w_{m i}\left(n-n^{\prime}\right) r_{i}\left(n^{\prime}\right), \quad(15) \\
1 \leq m \leq M
\end{array}
$$

where

$$
r_{i}\left(n^{\prime}\right)=\sum_{l=1}^{L} v_{i}(l) p\left(n^{\prime}, l\right)
$$

Eqn. 15 is in the form of multi-channel convolution, and Eqn. 16 is in the form of projection. Therefore, we obtained the desired form of the measurement relation in which the unknown property $p(n, l)$ is related to the measurements by the separable two stage operations of projection and convolution. Hence, inversion operation involves the inversion of these stages: multi-channel deconvolution followed by back-projection. These two stages of the inversion can be written as:

$$
\hat{r}_{i}(n)=\sum_{m=1}^{M} h_{i m}(n) * g_{m}(n), \quad 1 \leq i \leq I
$$

where $*$ denotes convolution operation, and

$$
\hat{p}(n, l)=\sum_{i=1}^{I} \hat{r}_{i}(n) v_{i}(l)
$$

In Eqn. 17 a set of multi-channel deconvolution filters are used to obtain the estimate $\hat{r}_{i}(n)$ of $r_{i}(n)$. Then, the estimate $\hat{p}(n, l)$ can be obtained by the back-projection step of Eqn. 18. As it can be immediately recognized, the critical part of the inversion is the deconvolution stage. One of the requirements in the design of the required deconvolution filters is that of robustness to the additive noise in the measurements. In this work, we used one such design procedure reported in [3]. It is important to note that, although the design of the deconvolution filters is computationally involved, it has to be done only once for a fixed data acquisition geometry. Once the deconvolution filters are computed and stored, the actual stages of inversion can be performed quite efficiently.

\section{Simulations}

The measurement setup depicted in fig. 1 was used for simulations. The sample material was chosen as aluminum $\left(\mathrm{V}_{l}=6420 \mathrm{~m} / \mathrm{s}, \mathrm{V}_{s}=3040 \mathrm{~m} / \mathrm{s}\right.$ [7]). The coupling fluid is water. The transducer is excited with a gated RF-pulse of frequency $1 \mathrm{MHz}$ and duration $40 \mu \mathrm{sec}$. Field generated by the transducer is propagated down to the object surface using angular spectrum decomposition. Field 
on the surface was assumed to directly couple to SAW. Along the x-axis, 256 field samples were taken with $d x=0.98 \mathrm{~mm}$, and there were 450 samples along the $y$-axis with $d x=1.2 \mathrm{~mm}$.

A sample reconstruction is shown in Fig. 3 . Three point scatterers are assumed to exist at grid points $(0,51),(0,90)$ and $(3,90)$. The $y$-axis of the image is expanded using the warping function.

\section{Conclusions}

In this work, it is shown that the measurement relation of the commonly used synthetic aperture data acquisition system can be put into a new form allowing to use a novel efficient regularized inversion algorithm. Simulations have shown that the inversion algorithm provides robust high resolution images.

\section{References}

[1] J. T. Ylitalo and H. Ermert, "Ultrasound synthetic aperture imaging: monostatic approach," IEEE Trans. Ultrason., Ferroelec., Freq. Contr., vol. 41, pp. 333-339, 1994.

[2] S. Bennett, D. K. Peterson, D. Corl and G. S. Kino, "A real-time synthetic aperture digital acoustic imaging system," in Acoustic Imaging, P. Alais and A. F. Metherell, Eds. New York: Plenum, 1980, vol. 10, pp. 669-692.

[3] O. Arrkan, "Regularized inversion of a twodimensional integral equation with applications in borehole induction measurements," Radio Science, vol. 29, pp. 519-538, 1994.

[4] N. Dunford and J. T. Schwartz, Linear Operators, New York: Wiley Interscience, 1964.

[5] Golub, G. H. and Van Loan, C. F., Matrix Computations, $2^{\text {nd }}$ ed., Baltimore: John Hoppkins Univ. Press., 1989.

[6] A. Atalar, "A backscattering formula for acoustic transducers," J. Appl. Phys., vol. 51, pp. 3093-3098, 1980.

[7] A. Briggs. Acoustic Microscopy. Oxford University Press, Oxford, 1992.

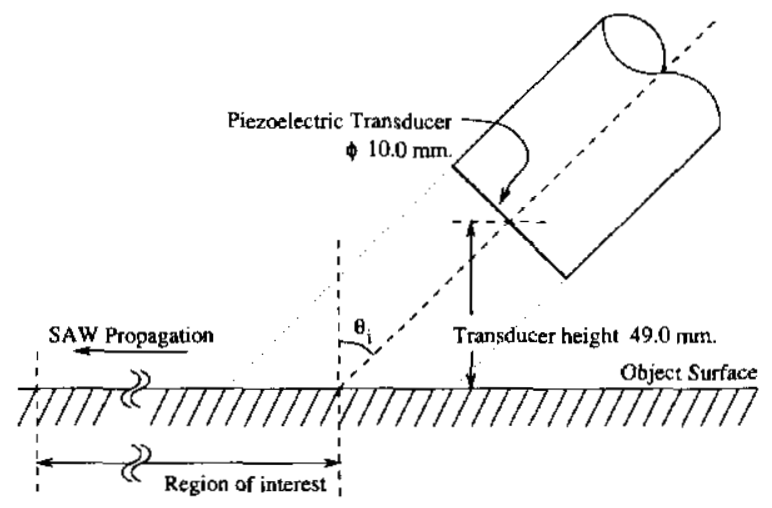

Figure 1: Measurement setup.

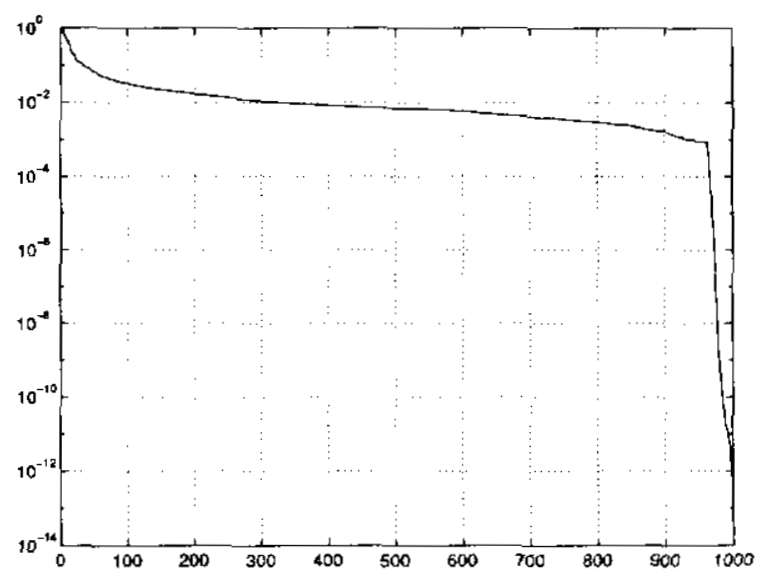

Figure 2: Singular values of the Hilbert-Schmidt decompositon of the auxilary function $Q\left(t_{1}, t_{2}\right)$.

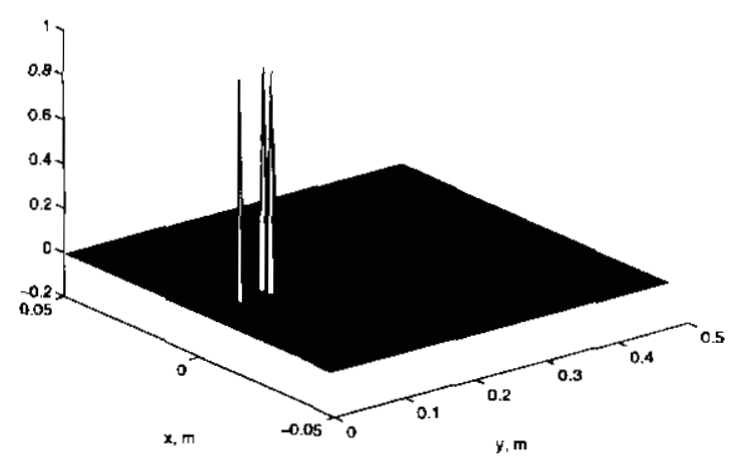

Figure 3: Sample reconstruction. 